\title{
Contact stresses in point frogs
}

\author{
Boris Glusberg ${ }^{1}$, Vadim Korolev $^{2}$, Olga Lokteva ${ }^{2}$, and Irina Shishkina ${ }^{2 *}$ \\ ${ }^{1}$ Railway Research Institute (JSC VNIIZhT), 3rd Mytischinskaya str., 10, Moscow, 129626, Russia \\ ${ }^{2}$ Russian University of Transport (MIIT), Chasovaya str. 22/2, Moscow, 125190, Russia
}

\begin{abstract}
The methodology for calculating the contact stress state for various contact types of frogs and wheel is presented. The magnitudes of contact stresses in the frogs can be calculated according to the HertzBelyaev method, taking into account certain evaluating factors.
\end{abstract}

\section{Introduction}

An increase in axial loads, train speeds, and railway load carrying capacity increases the failure of frogs due to contact fatigue damage $[1,2]$. For that reason, about $25 \%$ of frogs that have not reached the limit of wear and tear are taken out of the track.

The contact durability of crosses is increased by hardening the metal, improving its structure, improving the geometry of work surfaces and by other methods $[3,4,5]$. In order to successfully solve this problem, it is necessary to know the magnitude of the contact stresses arising in the frogs from the wheels of the rolling stock.

The methods of measuring contact stresses of the frogs in the track have not yet been developed. Therefore, researchers involved in improving the contact-fatigue strength of frogs have to use the Hertz-Belyaev theory and other calculation methods.

The contact of the wheel and the frog belongs to the class of problems, for which the premises of the Hertz-Belyaev theory are not fully satisfied. There are such features as the commensurability of the radii of the contacting bodies; the proximity of the contact pad to the edge of the side edge; the commensurability of the contacting body radius and the width of the contact pad, etc. $[6,7]$. Contact stress epurs for this class of contact issues are similar to epurs obtained according to the Hertz-Belyaev theory. However, a large mismatch can be obtained in the numerical values of the stresses. It is possible to take into account these features with the help of a system of evaluation coefficients for the calculated values, which can be obtained according to the Hertz-Belyaev theory. Let us consider their definition.

\section{Research technique}

The study of contact stresses in the frogs was carried out experimentally using models and by the photoelasticity method, which allows accurately obtaining the stress values and studying the nature of their distribution inside the loaded element.

The stress state of any loaded element depends on the magnitude of the load, the geometric shapes and sizes of the element, its elastic characteristics and the method of the

* Corresponding author: $\underline{\text { shishkinaira@inbox.ru }}$ 
load application $[8,9,10]$.

The contact pads of the wheels and frogs in the rolling zone have the shape of ellipses that are elongated along the element. According to experimental data, the average width of the contact pads in the rolling zone varies between $5.4-6.7 \mathrm{~mm}$, and the minimum width varies between $2.0-4.0 \mathrm{~mm}$. With such a width of the contact pads, the eccentricities of contact ellipses are in the range $e=0.900 \div 0.999$. In order to obtain statistics on $e$, the sizes of contact ellipses of new and middle-worn wheels and frogs were calculated, with wear in the $40 \mathrm{~mm}$ core section from 0 to $12 \mathrm{~mm}$. The diameters of full-sized crosses taken by a profilograph were used. The diameter of the middle-worn wheel is taken according to the mass measurement of the wheels. A total of 120 different contact options were calculated.

The calculated values of the eccentricity of contact ellipses were $e=0.82 \div 0.999$, and only in eight contact variants there were areas with $e<0.9$. For values of $e \geq 0.9$, the stress state parameters in the contact of the wheel and the frog differ little from those obtained from the condition of contact in an infinite strip $(e=1.0)[11,12,13]$. So, it can be obtained that the magnitudes of the highest stresses calculated within this limit of the change in eccentricities $(0.9 \leq \mathrm{e} \leq 1.0)$ differ by no more than $4.8 \%$. The ratio of the depth of points with the highest tangential stresses values to the width of the contact area differ by no more than $12.8 \%$.

The greatest contact stresses in all cases arise in the cross section passing through the center of the contact ellipse. When contacting along an infinite strip, the element in the contact zone operates in a state of plane deformation $[14,15]$.

Thus, in most cases, the metal of the frogs in the contact region of the rolling zone is undergoing a deformation, which is close to plane (i.e., deformations along the axis perpendicular to the considered section can be neglected).

Elastic characteristics are not included in the equilibrium equations of a stressed element. In a plane problem of six equations, only one equation for the compatibility of deformations remains:

$$
\frac{d^{2} \varepsilon_{z}}{d y^{2}}+\frac{d^{2} \varepsilon_{y}}{d z^{2}}=\frac{d^{2} \gamma_{z y}}{d z d y}
$$

where $\gamma_{z y}, \varepsilon_{z}, \varepsilon_{y}$ are relative strains; $\mathrm{z}$ is the axis directed normal to the contacting bodies; $y$ is the axis perpendicular to the $\mathrm{z}$ axis in the plane of the cross section.

The strain compatibility equation can be obtained through stresses:

$$
\left(\frac{\partial^{2}}{\partial z^{2}}+\frac{\partial^{2}}{\partial y^{2}}\right)\left(\sigma^{z}+\sigma^{y}\right)=-\frac{1}{1-\mu}\left(\frac{\partial Z}{\partial z}+\frac{\partial Y}{\partial y}\right)
$$

where $\mathrm{Z}, \mathrm{Y}$ - bulk forces; $\mu$ - Poisson's ratio.

In general case, the Poisson's ratio is included in the strain compatibility equation and affects the stress distribution within the loaded element.

In the problem under consideration, the bulk load is only the dead weight of the frog, which does not depend on the coordinates. Therefore, $\frac{\partial Z}{\partial z}=0$ and $\frac{\partial Y}{\partial y}=0$. The strain compatibility equation is independent of the Poisson ratio, i.e.

$$
\left(\frac{\partial^{2}}{\partial z^{2}}+\frac{\partial^{2}}{\partial y^{2}}\right)\left(\sigma^{z}+\sigma^{y}\right)=0
$$

Since the frog sections under consideration are simply connected (there are no internal cavities), both conditions of the Levy-Mitchell theorem are satisfied for the conditions of the frog contact issue. Therefore, distribution of contact stresses in the frog does not depend on elastic characteristics [16]. This allows replacing the study of a plane strain state with a technically simpler study of a plane stress state, since the compatibility equations of the deformations of these two states only differ in a factor depending on the Poisson's ratio.

The photoelastic method is used to study the stress state of transparent models made of a special piezoelectric material.

If it is possible to establish a one-to-one correspondence between the stress state of the 
model and the studied full-sized element using the theory of modeling and then study the stress state of the model, then it is possible to carry out a transformation allowing to study stress state of the full-sized element $A^{\mathrm{H}}=m_{A} A^{\mathrm{M}}$, where $A^{\mathrm{H}}$ and $A^{\mathrm{M}}$, are the corresponding scalars, vectors or tensors for full size and for model; $m_{A}$ is the transformation that determines compliance.

In the problem under consideration, the stress distribution in the contact region does not depend on the elastic characteristics of the elements material. Therefore, establishing a correspondence between the model and full-size reduces to establishing a geometric similarity between them $[17,18]$.

Contact stresses in contacting bodies substantially depend on the curvature value of their surfaces at the contact point. Therefore, in order to simplify the study, the geometric scale of the model was adopted as 1:1. In order to load models of the frogs with the wheel models, a special loading device was made, which was placed in the load frame of the installation and allowed varying loads in a wide range. Calibration and pricing of the model strip was carried out on a disk with a diameter of $60 \mathrm{~mm}$. The disc is made of the same plate, from which the frog models were made of $[19,20]$.

A total of 87 different types of contact between the frog and the wheels were studied, 40 of them for the frog cross section with a core width of $35 \mathrm{~mm}$ and 47 for the cross section with a core width of $20 \mathrm{~mm}$. The frog models were made with transverse surface profiles corresponding to the new frog and worn one, with the maximum wear and tear limit. The diameters of the wheel models were taken with the following profiles: new, medium-worn, extremely worn out and with stepped section. Various forms of contact were investigated depending on the position of the wheels in the gutter of the frog. The strip patterns (Fig. 1) were photographed on photographic plates, and the isocline pictures were drawn directly on a sheet of paper fixed on the installation screen.
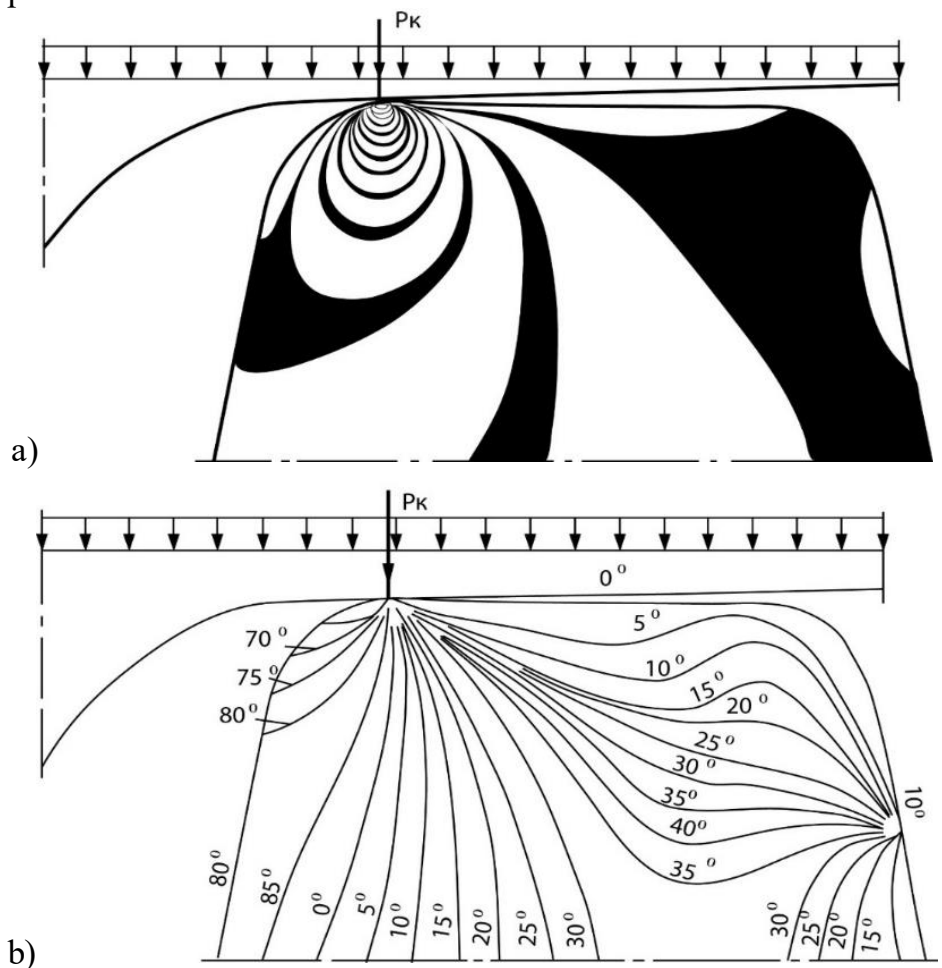

Fig. 1. The strip pattern (a) and isocline (b) in the core model of an unworn frog in a $35 \mathrm{~mm}$ wide section from the impact of a new wheel 
The highest tangential stresses in the model plane were obtained directly from the strip pattern (Fig. 2), and the normal stresses $\sigma_{z}$ and $\sigma_{y}$ were obtained by the separation method. Stresses were separated by numerical integration of the equilibrium equations. Normal stresses $\sigma_{x}$ in the plane perpendicular to the plane of the model were determined by the formula $\sigma_{x}=\mu\left(\sigma_{z}+\sigma_{y}\right)$.

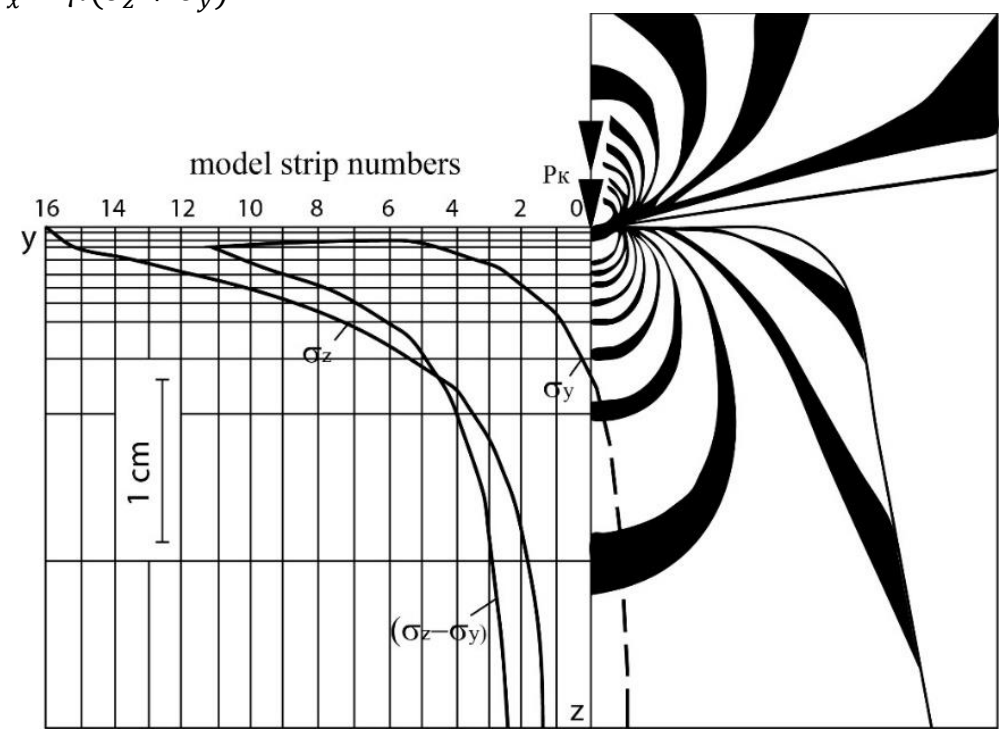

Fig. 2. The strip pattern and the epurs of contact stresses obtained from it in the model of the frog core with a section of $20 \mathrm{~mm}$

In order to verify the accuracy of the experiment, contact stresses were studied for the case of disk pressure $d=60 \mathrm{~mm}$ per half-plane. The difference in the values of the stresses, which were obtained experimentally and theoretically, did not exceed 3-6\% (a smaller figure refers to the maximum values of the stresses) [21, 22, 23].

\section{Results and discussion}

For all contacting options, the results were compared with the stresses obtained by calculation using the Hertz-Belyaev theory. The correction coefficients $k=\frac{\sigma^{E}}{\sigma^{C}}$ were calculated, where $\sigma^{E}$ are the stresses obtained experimentally by the photoelasticity method; $\sigma^{C}$ are the stresses obtained by calculation by the Hertz-Belyaev method.

The values of $k$ ranged from 0.62 to 1.01 , however, for each specific case, the range was small (up to 5\%). The average values of $k$ are shown in Fig. 3. The type of stress epurs coincided in almost all cases. Thus, the values of contact stresses in the frogs can be obtained by using the formulas $\sigma=k_{\sigma} \sigma^{*}$ and $\tau=k_{\tau} \tau^{*}$, where $k_{\sigma}, k_{\tau}$ are the transition coefficients; $\sigma^{*}, \tau^{*} *$ are the corresponding stresses obtained by calculation according to the Hertz-Belyaev theory.

In the case of contact between the frog and the wheel having a stepped tyre wear, the stresses could not be measured, since residual plastic deformations occurred even with a small initial load in the frog model. Nevertheless, this phenomenon in itself allows concluding that such wheels can significantly damage the frogs lying in the way.

The methodology described above can be used to calculate the contact stress state for various contact variants of frogs and wheels. This will allow determining the location of the most stressed points in the contact region of the rolling zone, in which microdefects of 
fatigue origin can arise, and also to predict the development directions of these defects.

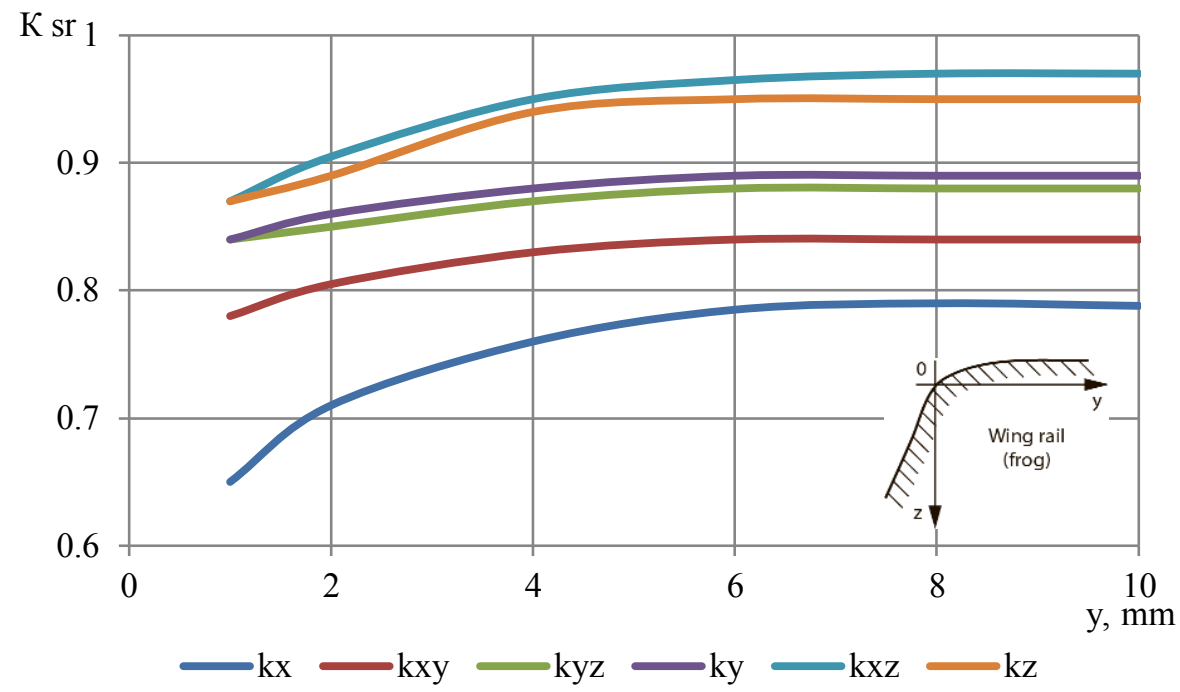

Fig. 3. Average correction factors for stress values, calculated according to Hertz-Belyaev theory

\section{Conclusions}

1. The numerical values of contact stresses in the frogs differ according to the values obtained by using the Hertz-Belyaev method by $0.62-1.01$ times.

2. The values of contact stresses in the frogs can be obtained by calculation according to the Hertz-Belyaev method, taking into account the correction factors obtained in this study.

\section{References}

1. B. Glusberg, V. Korolev et al. MATEC Web of Conferences, 239, 01054 (2018) DOI: 10.1051/matecconf/201823901054

2. B. Glusberg, V. Korolev et al. E3S Web of Conferences, 138, 01017 (2019) DOI: $10.1051 / \mathrm{e} 3$ sconf $/ 201913801017$

3. E. Gridasova, P. Nikiforov, A. Loktev et al. TransSiberia 2019: VIII International Scientific Siberian Transport Forum, 559-569 DOI: 10.1007/978-3-030-37916-2_54

4. A.A. Loktev, V.V. Korolev, E.A. Gridasova. IOP Conf. Series: Materials Science and Engineering, 687, 022036 (2019) DOI: 10.1088/1757-899X/687/2/022036

5. I. Shishkina. TransSiberia 2019: VIII International Scientific Siberian Transport Forum, 834-844, DOI: 10.1007/978-3-030-37916-2_82

6. B. Glusberg, A. Savin et al. Advances in Intelligent Systems and Computing, 982, 556570 (2020) DOI: 10.1007/978-3-030-19756-8_53

7. B. Glusberg, A. Savin et al. Advances in Intelligent Systems and Computing, 982, 571587 (2020) DOI: 10.1007/978-3-030-19756-8_54

8. V. Korolev. TransSiberia 2019: VIII International Scientific Siberian Transport Forum, 621-638 DOI: 10.1007/978-3-030-37916-2_60

9. A.V. Savin, V.V. Korolev, I.V. Shishkina. IOP Conf. Series: Materials Science and Engineering, 687, 022035 (2019) DOI: 10.1088/1757-899X/687/2/022035 
10. A. Savin, O. Suslov et al. TransSiberia 2019: VIII International Scientific Siberian Transport Forum, 648-654 DOI: 10.1007/978-3-030-37916-2_62

11. A. Loktev, V. Korolev, I. Shishkina et al. TransSiberia 2019: VIII International Scientific Siberian Transport Forum, 2, 1116 (2020) DOI: 10.1007/978-3-030-37919-3

12. A. Loktev, V. Korolev et al. IOP Conf. Series: Materials Science and Engineering, 463, 032019 (2018) DOI: 10.1088/1757-899X/463/3/032019

13. A. Loktev, V. Korolev et al. Vestnik of the Railway Research Institute, 77(6), 331-336 (2018) DOI: 10.21780/2223-9731-2018-77-6-331-336

14. A. Loktev, V. Korolev et al. Vestnik of the Railway Research Institute, 77(2), 77-83 (2018) DOI: 10.21780/2223-9731-2018-77-2-77-83

15. A. Loktev, V. Korolev et al. IOP Conf. Series: Materials Science and Engineering, 463, 032018 (2018) DOI: 10.1088/1757-899X/463/3/032018

16. V. Vorobyov, A. Manakov, A. Reger, I. Tanaino, MATEC Web of Conferences 216, 02009 (2018). DOI: 10.1051/matecconf/201821602009

17. A. Manakov, A. Abramov, A. Ilinykh, V. Aksenov, MATEC Web of Conferences 239, 04001 (2018). DOI: 10.1051/matecconf/201823904001.

18. A. Loktev, V. Korolev et al. Advances in Intelligent Systems and Computing, 982, 325-336 (2020) DOI: 10.1007/978-3-030-19756-8_30

19. B. Glusberg, A. Loktev et al. Advances in Intelligent Systems and Computing, 982, 337-345 (2020) DOI: 10.1007/978-3-030-19756-8_31

20. A. Lyudagovsky, A. Loktev, V. Korolev et al. E3S Web of Conferences, 110, 01017 (2019) DOI: $10.1051 / \mathrm{e} 3$ sconf/201911001017

21. A. Savin, A. Kogan et al. International Journal of Innovative Technology and Exploring Engineering, 8(7), 2325-2328 (2019)

22. A. Savin, V. Korolev et al. TransSiberia 2019: VIII International Scientific Siberian Transport Forum, 797-808 DOI: 10.1007/978-3-030-37916-2_78

23. V. Korolev. TransSiberia 2019: VIII International Scientific Siberian Transport Forum, 621-638 DOI: 10.1007/978-3-030-37916-2_60 